\title{
Remote Sensing Data Reveals Eco-Environmental Changes in Urban Areas of Klang Valley, Malaysia: Contribution from Object Based Analysis.
}

\begin{abstract}
Understanding the growth and changes in urban environments are the most dynamic system on the earth's surface is critical for urban planning and sustainable management. This study attempts to present a space-borne satellite-based approach to demonstrate the urban change and its relation with land surface temperature (LST) variation in urban areas of Klang valley, Malaysia. For this purpose an object-based nearest neighbour classifier (S-NN) approach was first applied on SPOT 5 data acquired on 2003 and 2010 and subsequently five land cover categories were extracted. The overall accuracies of the classified maps of 2003 and 2010 were $90.5 \%$ and $91 \%$ respectively. The classified maps were then used as inputs to perform the post classification change detection. The results revealed that the post-classification object-based change detection analysis performed reasonably well with an overall accuracy of $87.5 \%$, with Kappa statistic of $0.81 \%$. The changes represented that the urban expanded by $10 \%$ over the period, whereas the urban expansion had caused reduction in soil $(1.4 \%)$ and vegetation $(11.4 \%)$, and growth in oil palm $(2 \%)$, and water $(0.7 \%)$. Additionally decision tree method was used to derive the surface heat fluxes from thermal infrared Landsat TM and ETM+bands. Subsequently, a comparison was made with classified result from SPOT 5 images. Results showed high correlation between urban growth and LST.
\end{abstract}

Keyword: Eco-environmental change, Remote sensing, GIS, Klang Valley, Malaysia 